\title{
Service user perspectives of community mental health services for people with complex emotional needs: a co-produced qualitative interview study
}

Kylee Trevillion ${ }^{1,2^{*}}$, Ruth Stuart ${ }^{1}$, Josephine Ocloo ${ }^{3,4}$, Eva Broeckelmann ${ }^{5}$, Stephen Jeffreys ${ }^{5}$, Tamar Jeynes ${ }^{5}$, Dawn Allen ${ }^{5}$, Jessica Russell ${ }^{5}$, Jo Billings ${ }^{6}$, Mike J. Crawford ${ }^{7}$, Oliver Dale ${ }^{8}$, Rex Haigh ${ }^{9}$, Paul Moran $^{10}$, Shirley McNicholas ${ }^{11}$, Vicky Nicholls ${ }^{6}$, Una Foye ${ }^{1}$, Alan Simpson ${ }^{12}$, Brynmor Lloyd-Evans ${ }^{6}$, Sonia Johnson ${ }^{6,11}$ and Sian Oram ${ }^{1}$

\begin{abstract}
Background: There is consensus that services supporting people with complex emotional needs are part of a mental health care system in which change is needed. To date, service users' views and co-production initiatives have had little impact on the development of interventions and care. This needs to change, and our paper evidences the experiences and perspectives of a diverse range of people on how community services can best address the needs of people with complex emotional needs.

Methods: A co-produced qualitative research study. Lived experience researchers led data collection and analysis. Individual interviews were conducted with 30 people across England who had a diverse range of experiences and perspectives of using community services for complex emotional needs. Participants were asked about their experiences of using community services for their mental health, and views on how community services can best address their needs. Thematic analysis was used to analyse the data.

Results: Participants reported some experiences of good practice but also of experiences of severely stigmatising interventions, a lack of effective support and service fragmentation. Relational Practice was identified as the central overarching theme and describes how community services can best support people with complex emotional needs. This approach involves care delivered in a non-stigmatising, individualised and compassionate way and care that is trauma-informed. It involves care that is planned collaboratively with service users to ensure their multiple needs are addressed in a flexible, holistic and consistent way which accounts for the long-term and fluctuating nature of their needs.
\end{abstract}

Conclusions: Relational practice approaches have potential to facilitate better community care for people with complex emotional needs. Research and service development are needed to examine how best to implement such

\footnotetext{
*Correspondence: kylee.trevillion@kcl.ac.uk

2 David Goldberg Centre, Institute of Psychiatry, Psychology \&

Neuroscience Kings College London, De Crespigny Park, Denmark Hill,

Room H3.06, London SE5 8AF, UK

Full list of author information is available at the end of the article
}

(c) The Author(s) 2022. Open Access This article is licensed under a Creative Commons Attribution 4.0 International License, which permits use, sharing, adaptation, distribution and reproduction in any medium or format, as long as you give appropriate credit to the original author(s) and the source, provide a link to the Creative Commons licence, and indicate if changes were made. The images or other third party material in this article are included in the article's Creative Commons licence, unless indicated otherwise in a credit line to the material. If material is not included in the article's Creative Commons licence and your intended use is not permitted by statutory regulation or exceeds the permitted use, you will need to obtain permission directly from the copyright holder. To view a copy of this licence, visit http://creativecommons.org/licenses/by/4.0/. The Creative Commons Public Domain Dedication waiver (http://creativeco mmons.org/publicdomain/zero/1.0/) applies to the data made available in this article, unless otherwise stated in a credit line to the data. 
approaches across the mental health service system. This work must be co-produced with people with relevant lived experience, their carers and the professionals who support them.

Keywords: Qualitative research, Personality disorders, Community mental health services, Co-production

\section{Background}

The focus of this paper is on community mental health service use among people with complex emotional needs (CEN). CEN refers to people who may have received a 'personality disorder' diagnosis and/or have used services for 'personality disorder', or who appear to have similar needs (e.g. related to repeated self-harm). Our team of experts by experience, experts by occupation (i.e. people who provide care/deliver support to service users) and health researchers recognise the considerable stigma attached to the label 'personality disorder', and the considerable associated harms identified by both service users and clinicians [1,2]. Indeed, significant critiques have been made of the 'personality disorder' diagnosis as stigmatising, misogynistic and associated with an absence of hope and of progress in the delivery of healthcare [3-6]. Though some service users find the term helpful in explaining the nature of their needs, and it has had a role in ensuring consistency in research, many people find it unhelpful and do not identify with it. With consideration of these ongoing and evolving debates, at the beginning of the project our team of experts came together to discuss and agree on a preferred alternative working term - complex emotional needs (CEN). In this paper, and in our study materials, we use the working term CEN which is also used by some mental health services in the UK. It is not our intention that complex emotional needs becomes a substitute diagnosis, but rather a description of a broad group of service users and survivors. We acknowledge its limitations, in terms of being rather over-general, and advocate for further coproduced work to develop new ways of describing this need and on the best way of assessing people with these clusters of need.

A recent systematic review examined the worldwide prevalence of CEN across 21 countries and estimated that up to $8 \%$ of community populations are affected [7]. Higher rates are found within community healthcare settings, with around a quarter of people accessing primary care services and half of people accessing outpatient mental health services identified as having CEN $[8,9]$. Evidence indicates that the prevalence of CEN in the general community is similar among men and women and as common among minority ethnic groups as majority groups $[10,11]$. Yet, in clinical populations the prevalence of CEN is lower among minority ethnic groups and among men. It is unclear whether these differences reflect a lower prevalence meeting diagnostic criteria or instead lower rates of service use and/or under-detection by services $[11,12]$.

People given a CEN diagnosis are found to be more stigmatised than people given other psychiatric diagnoses and this stigmatisation has led people to experiencing exclusion or limited attention from mental health services [13]. Several reasons for this increased stigma are reported, including a lack of knowledge about this mental health need among the general public and staff who support people with CEN [14]. This lack of knowledge is compounded as people hold varied and implicit understandings about the concept of personality and this can influence the nature of stereotypes they hold about a person who is given a 'personality disorder' diagnosis [15]. The concept of mental illness infers that symptoms are extrinsic to the person but it is argued that distinctions between the person and their mental health needs are blurred in diagnostic classifications of CEN [15]. This may provide some insight into why people diagnosed with CEN are viewed as less ill and more accountable for their behaviours compared to people diagnosed with other mental health needs [16]. Research indicates that staff who support people with CEN can experience negative feelings of frustration, incompetence and helplessness in response to some of the behaviours expressed by people with CEN (e.g. anger and self-harming behaviours) and this may lead them to adopt negative and avoidant attitudes [2].

In light of the above, it is perhaps unsurprising that mental health services are found to marginalise service users with CEN and, compared with other mental health conditions, the provision of timely, well-resourced interventions and good quality care for this service user population appears to lag behind [2, 17-19]. Our recent meta-synthesis of the international evidence on service users' experiences of mental health services identified several areas for which there is a strong consensus on what kind of care is needed [1]. These include providing holistic support (i.e. support that addresses service users' psychological, social, and physical needs), delivered by skilled and compassionate staff who understand the need for a long-term perspective on intervention. Our complementary meta-synthesis of international clinician perspectives highlights that some staff, especially in generic mental health services may lack the knowledge and skills to effectively support people [2]. Access to specialist 
services and longer-term interventions are also reported to be impeded by a lack of clear referral pathways and accessible services for people at various stages in recovery journeys [2]. Clinicians call for better organisational support, more joint-working practices and clinical supervision to assist them in delivering better care to service users [2].

Given the high levels of need within community services (defined in this study as publicly-funded primary care services, non-specialist secondary mental health services, specialist community 'personality disorder' services and non-profit community organisations and networks that work with people with CEN, and excluding community forensic mental health services) and the considerable variability in service quality, a growing number of international policy guidelines are intended to improve and enhance community care for people with CEN [20, 21]. Yet, the data sources used to develop many of these guidelines fail to incorporate the views and perspectives of service users and the family and friends who support them [20, 22]. In addition, most research on care models for people with CEN focus on psychological interventions, especially those intended to reduce self-harm [23]. They ignore the more general principles of how to provide good care and ensure peoples' needs are met across healthcare service systems. In this co-produced qualitative study, our team of researchers and clinicians, including several with relevant lived experience, aimed to identify best practice in community interventions and support for people with CEN from the perspective of services users. This study contributes to foundations for intervention development and service improvements that are informed by service user perspectives and priorities. The study objectives were:

1. To explore the experiences of adults with CEN in using community services for their mental health in a range of English service settings, including National Health Service (NHS) and voluntary sector services

2. To explore the views of adults with CEN about how community services can best meet their needs

\section{Methods}

We followed CORE-Q reporting guidelines for qualitative research [24].

\section{Study design}

This study is a co-produced qualitative research study. Co-production in research can be defined as research which brings together experts by experience, experts by occupation and researchers who work together, sharing power and responsibility to form equitable partnerships, on a study from the beginning to the end $[25,26]$. Coproduction approaches differ but they all seek to move beyond simply consultation and collaboration exercises $[26,27]$. Co-production approaches take the position that those affected by the research have skills and knowledge of equal importance to researchers and are best placed to design and deliver it. In co-produced research studies, all members of the team work towards a shared understanding, there is joint ownership over key decisions, everyone on the team is recognised as an asset and their individual contributions are recognised [25].

\section{Research team}

This study was co-produced from inception, design, and delivery by nine members of the Mental Health Policy Research Unit (KT, RS, JO, UF, SJo, AS, BLE, VN, SO), six experts by experience (SJe, EB, TJ, DA, JR and Gabriella Clarke) and nine experts by occupation (OD, PM, MC, RH, SMc, JB, Alison Bearn, Brian Solts, Penny Bennett). The Mental Health Policy Research Unit (MHRPU) supports the development of nationwide planning of mental health services through evidence-based research. A co-production group was established at the outset of the study and group meetings occurred approximately every quarter between July 2019 and December 2020; work on co-producing this manuscript was conducted via videocalls and emails, through early 2021, with all manuscript authors. Group team meetings were chaired by one of the experts by occupation (SMc) and all group members were invited to every team meeting. Co-production work ran throughout the course of the study (e.g. via coproduction of the study protocol, interview topic guide and analyses). Experts by experience led the research interviews; five of the six experts by experience (SJe, EB, TJ, DA and JR) conducted 24 of the 30 interviews (the remaining six were conducted by a health researcher $(\mathrm{KT})$ ). Experts by experience led the coding of interview transcripts; four experts (EB, SJe, DA, JR) coded most of the interview transcripts (17/30 interviews) and the remaining 13 transcripts were coded by health researchers (Nafiso Ahmed, RS, KT, UF, Norha Vera San Juan). Data analyses were jointly undertaken by all manuscript authors, with key themes established through consensus discussions. Researchers in the MHPRU (VN, KT) and an expert qualitative researcher (Dr Nicola Morant) provided training on conducting qualitative interviews (including role-play exercises and an observation of a live interview) and training on analysing qualitative research with the lived experience members of the team. An MHPRU researcher (Sarah Carr) supported KT and $\mathrm{VN}$ in preparing a protocol around promoting the emotional well-being of lived experience researchers who conducted the research interviews. One of the experts by 
occupation (JB, a clinical psychologist) offered the lived experience researchers individual support sessions about the emotional content and impact of the work throughout the interview period. Two health researchers (KT; JO - supported by RS) led on setting up the study, including obtaining ethical approvals/amendments and establishing data management systems. The two researchers (KT; JO) also coordinated the overall study progress, including coordinating co-production group meetings, identifying and engaging recruitment sites/networks, leading on recruitment processes and procedures (including purposive sampling using a culturally competent approach, led by JO) and coordinating interview procedures. Two health researchers adapted the study documentation and interview procedures following the pandemic (JO, RS). Of the paper authors, seven are male, and two have Black or mixed heritage backgrounds.

\section{Study sample}

People were eligible for the study if they were: adults (aged 18 or above) who had received a diagnosis of a 'personality disorder' or who self-identified as having difficulties that may result in a 'personality disorder' diagnosis or in using CEN services (e.g. recurrent self-harm, other impulsive behaviour); who may have used community services for their mental health; who could undertake an interview in English; who had capacity to consent to the research. We recruited 30 people and aimed to ensure our sample represented a full range of characteristics with respect to age, gender, sexuality, ethnicity, geographical location in England and type of community service use. We did this by asking individuals who were potentially interested in participating to describe their characteristics and used this information to purposively select a group of people who presented a diversity of characteristics. We kept an ongoing record of the key demographics of people we interviewed and reviewed this routinely, including during working group meetings, to check we were accessing a range of experiences and views from a diverse group of participants. We adopted an intersectional (i.e. recognizing individuals' intersecting identities including race, class, gender, sexuality, disability) and culturally competent (i.e. considering cultural identity and context) approach to recruitment to capture a diversity of perspectives.

\section{Study definition}

We defined community services as: (1) publicly-funded primary care services, which provide the first point of contact in the UK healthcare system (e.g. general practitioner services); (2) publicly-funded non-specialist secondary mental health services (e.g. community mental health teams) and specialist community 'personality disorder' services, which are accessed via referral from primary care and generic mental health services; (3) nonprofit community organisations and networks whose remit involves face-to-face work with people with CEN. Community forensic mental health services fell outside our study definition of community services.

\section{Recruitment}

Participants were recruited from voluntary sector organisations for people with mental health problems (e.g. National Survivor User Network), including those for Black communities and Lesbian, Gay, Bisexual, Transgender, Queer/Questioning people (LGBTQ+) and relevant online social media networks (e.g. the Mental Health Policy Research Unit, other personal and institutional Twitter accounts, the Mental Elf Twitter account, Facebook accounts on mental health). We used advertisements to recruit people and developed these to promote engagement of under-represented groups (e.g. including images of Black men and women and rainbow symbols for LGBTQ+ people). People interested in the study could either contact the research team directly, using the contact information on the advert, or ask that their information be passed on to the research team via the network managers/coordinators. One of the study researchers (KT or JO) had an initial conversation with people who were interested in the study, to establish whether they met eligibility criteria and to ensure diversity was achieved in the sample of people who were interviewed. Eligible participants were sent copies of the Participant Information Sheet at least $24 \mathrm{~h}$ before the interview. Participants were offered the option of being interviewed alone or with someone else present with them at the interview (e.g. a close friend or family member). Most participants $(n=24)$ were interviewed by the experts by experience members of the study team (SJe, EB, TJ, DA and JR); a health researcher (KT) conducted the remaining interviews $(n=6)$.

\section{Data collection}

Semi-structured individual research interviews were conducted between July 2019-October 2020 (N.B. the study was suspended between March and July 2020 in response to the COVID-19 pandemic). Interviews were guided by a topic guide co-developed by the study working group (please contact KT for further information about the topic guide). Before COVID-19, participants were given the option of being interviewed by a researcher either face-to-face, by Skype or telephone. Face-to-face research interviews were conducted within university settings. During COVID-19, with social distancing requirements in place, all interviews were conducted remotely using MS Teams or Zoom video-conferencing software; 
interviews were conducted by a researcher and a facilitator who was responsible for the interview recording. Informed consent was obtained from all participants prior to the interview, either in written form or verbally recorded. All interviews were recorded (either on an encrypted digital recorder or laptop) and were transcribed verbatim. The length of interviews varied from 30 to $100 \mathrm{~min}$.

\section{Analysis}

Thematic analysis (Braun and Clarke 2013) was used, and the data managed in NVivo Pro V12 [28]. Six key steps were undertaken. The first step, familiarisation with the data, involved detailed readings of the interview transcripts by researchers, who made initial reflections/ notes about the narratives. The second step, generation of initial codes, involved line-by-line open coding of each transcript. The team first did this as a group, using one transcript to establish an initial coding frame. The remaining transcripts were then shared out among researchers to complete the third, fourth and fifth steps of the analyses, involving searching for, reviewing, and defining the arising themes; the coding frame was revised and updated accordingly. The sixth step involved categorisation of data into a final set of themes which were conceptualised in a thematic map.

\section{Results \\ Sample}

We recruited 30 adults with diverse characteristics of age, ethnicity, geographical location and use of community services. Although we aimed to recruit some people who had not received a 'personality disorder' diagnosis, all participants had received a diagnosis at some point in their life. Some diversity was achieved with gender and sexuality (See Table 1).

\section{Findings}

Results are reported in line with the thematic conceptual map (Fig. 1), which presents the themes encapsulating how community services can best support people with CEN. The central theme in the map - Relational Practice - ties together all the other themes which describe how the approaches of individual staff and organisational structures/practices can promote positive therapeutic relationships and facilitate consistent, holistic and personalised care.

\section{Understanding}

The theme Understanding describes the need for community services to improve staff knowledge about and responses to CEN, as illustrated below.

\section{Staff knowledge about CEN}

Participants wanted to be supported by staff who were knowledgeable about their CEN and how to effectively support people with these needs:

"Having... the right workforce I think is really important. Even before you get to what services should be in place and where should they be, there is a how they should be operated, and that should be from... well informed practitioners" (participant 213, female, 35-44, White British, East Midlands)

Yet many participants described experiences of being seen by staff in non-specialist community mental health services who were not knowledgeable about how to support someone with CEN:

"It's not a well understood condition, either by the general public or by medical professionals. I think that's obviously a huge weakness, that people just don't know about or understand it" (participant 105, female, 35-44, White British, London)

\section{Non-stigmatising attitudes and behaviours}

Several participants reported stigmatising attitudes among some staff, with respect to the diagnostic label 'personality disorder' and how it is often perceived, including being seen as someone who cannot be helped, being de-personalised or being viewed as a potential trouble-maker. These experiences could be pathologising and harmful. Examples were seen across generic mental health and voluntary sector services:

"People still have this attitude that basically there's a group of people who are just impossible to work with and who will sabotage whatever you do" (participant 212, female, 45-54, White British, Yorkshire \& Humber)

"There is a disparity in services, but I think that is due to either the stigma placed on people as they enter the door, or whom they come across and how they are perceived...I think there is that, sort of, dismissal of who you are, and not seeing the person as a person. They just see the diagnosis" (participant 210, female, 25-34, Black British Caribbean, London).

Linked to this, participants commented that some staff view the needs of people with CEN as too challenging and so adopt dismissive or rejecting attitudes:

"It was really quite detrimental and actually harming when, rather than just saying, "We're finding this hard to deal with....It felt like I was 
Table 1 Study Sample Characteristics

\begin{tabular}{|c|c|c|}
\hline Primary sampling criteria & Category & $\mathbf{N}$ \\
\hline \multirow[t]{2}{*}{ Current diagnoses $^{\mathbf{a}}$} & Personality disorder & 29 \\
\hline & Complex post-traumatic stress disorder & 1 \\
\hline \multirow[t]{3}{*}{ Service use } & Specialist & 16 \\
\hline & Non-specialist & 10 \\
\hline & Unclear if specialist or non-specialist & 4 \\
\hline \multirow[t]{7}{*}{ Age } & $18-24$ & 3 \\
\hline & $25-34$ & 8 \\
\hline & $35-44$ & 8 \\
\hline & $45-54$ & 4 \\
\hline & $55-64$ & 5 \\
\hline & $65-74$ & 1 \\
\hline & Information not available & 1 \\
\hline \multirow[t]{2}{*}{ Sex } & Female & 24 \\
\hline & Male & 6 \\
\hline \multirow[t]{3}{*}{ Sexuality } & Heterosexual & 12 \\
\hline & Bisexual / Gay / Lesbian & 6 \\
\hline & Information not available & 12 \\
\hline \multirow[t]{5}{*}{ Ethnicity } & Black British /Caribbean & 2 \\
\hline & British Asian / Indian & 3 \\
\hline & Chinese & 4 \\
\hline & Mixed Race & 2 \\
\hline & White British / English / Other & 19 \\
\hline \multirow[t]{8}{*}{ Region of England (ONS classifications) } & North West & 2 \\
\hline & North East & 1 \\
\hline & Yorkshire / Humber & 2 \\
\hline & West Midlands & 2 \\
\hline & East Midlands & 3 \\
\hline & South West & 2 \\
\hline & London & 17 \\
\hline & South East & 1 \\
\hline
\end{tabular}

a Diagnoses included: anxious avoidant personality disorder, borderline personality disorder, emotionally unstable personality disorder, narcissistic personality disorder, obsessive compulsive personality disorder, paranoid personality disorder and schizotypal personality disorder. Some participants also received earlier/ concurrent diagnoses such as adjustment disorder, autism, bipolar disorder, depression, anxiety, eating disorder, or been mis-diagnosed with bipolar, depression or schizophrenia. Some had their type of 'personality disorder' diagnosis changed or replaced by complex post-traumatic stress disorder

being blamed for the fact that my needs couldn't be met" (participant 212, female, 45-54, White British, Yorkshire \& Humber).

These perceptions resulted in some participants being denied access or turned away from a range of mental and physical health services:

"My experience of services is largely one of being dismissed or discriminated against on the basis of my diagnosis. I've had that from all kinds of people, from dieticians, to psychiatrists, to psychotherapists" (participant 105, female, 35-44, White British, London).
The impact of these negative experiences left many participants feeling unheard and for a few it led them to disengage with services, as they felt it was causing them emotional harm:

"I didn't feel my voice was being heard. I actually broke down contact with them because I thought they were making me worse. I just thought I could live it out by myself" (participant 201, female, 35-44, Chinese, London).

Distinctions in how services responded to participants were made between generic mental health and specialist 'personality disorder' services: 


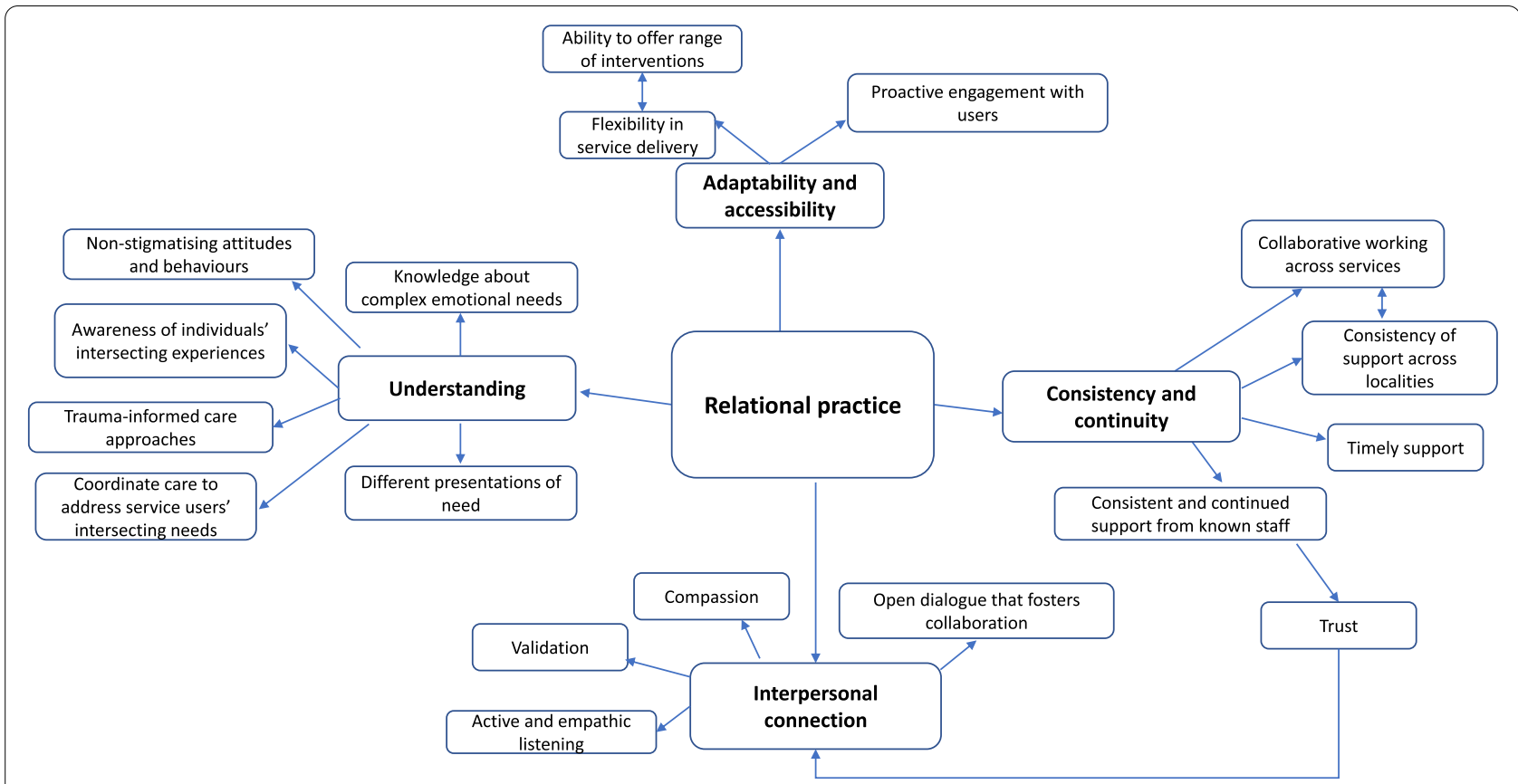

Fig. 1 Conceptual map

"Because they are specialist services you get less of the stigma, I think, than you do in the general CMHT...they [specialist services] know...the things that you are likely to struggle with, but you still feel like an individual...rather than..."You are this kind of person."” (participant 105, female, 35-44, White British, London).

\section{Awareness of individuals' intersecting experiences}

Participants spoke of the importance of staff educating themselves about the intersecting identities that service users may have and how these may impact on an individual's mental health:

"I am not expecting my therapist to have the same sexuality as me, but just being aware of the barriers, or the bi-phobia that a bi person can face, being aware of the different phobias" (participant 210, female, 25-34, Black British Caribbean, London).

"Having cultural intelligence and cultural awareness...so I am not having to explain why when somebody said something did it hurt me...I need to acknowledge how it hurt me, and sit with that, versus feeling like I'm having to convince somebody that my pain is justified" (participant 210, female, 25-34, Black British Caribbean, London).
Several participants reported experiences of discrimination connected to intersecting identities such as ethnicity, sexuality, age, class, physical appearance, and mental health need:

"It's to do with your ethnicity, you know, even your body size, your age, all sorts make you even less likely to be listened to. That's why I don't like telling my age and I don't like it when people ask about my ethnicity because they use all these things against you." (participant 203, female, Mixed Race, North West).

"Maybe it's to do with being Black and just not being seen as trustworthy or being seen as, if I'm getting upset, as being aggressive when context isn't taken into account. I feel like a lot of the stuff that's happened wouldn't have happened if I hadn't been Black" (participant 215, female, 25-34, Black British, London).

"Describing the text of a letter written by a community service* "IIt was] totally divorced from the context..."So, "[participant] is very angry and volatile." Not, "[participant] is very angry and volatile because he lives month by month and will he be able to cover the rent?... and he's really trying his hardest to get back to some so-called normality. That's why he's angry and volatile because he's getting no help 
and support from anyone"” (participant 111, male, 55-64, White Other, London).

Some participants spoke of needing to educate services about their intersecting identities and how they adversely impacted on their mental health:

"A huge part of my therapy became about me educating my therapist about a lot of the different things I had faced, or why it traumatised me, or why it affected me, because culturally we were different; ethnically we were different; different backgrounds, working class" (participant 210, female, 25-34, Black British Caribbean, London).

\section{Trauma-informed care}

Participants described the benefit of receiving traumainformed care [29] and called for wider adoption of this approach across community services. They described trauma-informed care as an understanding among staff that many people have developed CEN in response to past traumatic experiences and that changes in their behaviours often represent emotional responses to trauma. It is an approach that demonstrates compassion and also seeks to ensure people feel safe (e.g. establishing clear communication channels with service users, being aware of the physical space of meeting rooms and whether they are comfortable, inviting and not overly clinical, asking people where they'd like to sit in a meeting room):

"After the first few sessions [trauma-informed therapy], I felt like an explosion of knowledge in my learning and understanding...It's very amazing" (participant 107, female, 35-44, White British, London).

"Trauma-informed care...it acknowledges that... many people that suffer from personality disorder have done so because of the presence of early or indeed subsequent trauma in their life...This is a far more sympathetic and less judgemental way to proceed, and I look forward to when it is more mainstream than it currently is at the moment" (participant 208, male, 65-74, White Other, London).

Several participants described how their experiences of trauma were unacknowledged or even dismissed by services and described how organisational practices could inadvertently mirror abusive experiences:

"I was told very clearly that my history of trauma wasn't relevant" (participant 213, female, 35-44, White British, East Midlands).
"We've had so much instability in our lives [people with complex emotional needs]... Then when you go into services and services are chaotic or unstable, they [service users] don't trust it" (participant 101, female, 18-24, White British, London).

Another participant described how a lack of staff awareness of service users' intersecting identities and non-adoption of trauma-informed care may result in misdiagnosis for some people:

"A few nurses have said to me there are some Black men that...go around, keep using services...It's clear that there is some trauma going on. But they've never had the diagnosis. Because they will get something else as the diagnosis or...seen as the perpetrator, not a victim" (participant 202, female, 35-44, Indian, London).

\section{Coordinate care to address service users' multiple needs}

Participants want services to recognise the impact of their individual, interconnecting needs on their mental health, including not only needs related to symptoms but also to social needs and wider problems in living:

"People [staff] will look at things like medication and therapy but life is much more than those two things. You know, how lonely people are...I think [services] needs to look at all elements of your life" (participant 209, female, 45-54, White English, West Midlands).

"I think the priority is definitely the practical stuff... when I was homeless that was the priority...I really wasn't in a place to be dealing with the kind of psychological stuff" (participant 104, female, 25-34, White British, Yorkshire \& Humber).

"Quite a lot of people have got financial problems, quite a lot of people have got housing problems. All these things are massively linked to mental health" (participant 107, female, 35-44, White British, London.

In many cases, service providers did not appear to consider these factors, and this meant the type of support offered to participants was insufficient:

"The trauma of trying to keep the roof over my head and literally staying alive and eating and trying my best to stay off drugs... Every day was like a battle for mental and physical survival. It's like none of those things actually matter...They never seem to be able to take these things into context of how this might affect you in your mental health" (participant 111, 
male, 55-64, White Other, London).

Some participants described positive experiences of staff across generic, specialist and voluntary sector services working proactively to understand and address the full range of their needs:

"She [care-coordinator] probably did more than any therapist I've had to turn me around... bringing to my attention educational courses, that she felt would assist me....then able to intervene with the Local Authority...to change some of the appalling living conditions that I was surviving in at the time" (participant 208, male, 65-74, White Other, London).

"The [voluntary sector service programme] did offer...support with practical stuff which was, like, around social activity and social prescribing...how to do stuff... [it] did actually help" (participant 105, female, 35-44, White British, London).

\section{Different presentations of mental health need}

Participants described how some services failed to identify and acknowledge the extent of their mental health need because of their physical and verbal presentations; seen as suggesting that they were better able to cope than was the case:

"The fact that I'm articulate and that I make eyecontact, that I dress and I wash, has been the biggest barrier to me to getting care...I said, "Look, I may not have gone out of the house for five days but because I have an appointment, I've done what most people would do. But that doesn't mean that I'm well....I knew they didn't get it" (participant 108, female, 35-44, White British, London).

Consequently, several participants needed to advocate for themselves when engaging with services, but this approach often reinforced the perception that they were better able to manage their distress than was the case:

"There is this mismatch, I think, between me and my presentation, and me saying, "This is my distress. I need some help with this"...because I am able to do that the expectations are really high...that I should be able to resolve that and then it leads to this tension" (participant 213, female, 35-44, White British, East Midlands).

\section{Interpersonal connection}

The theme Interpersonal Connection describes the need for staff to adopt approaches which facilitate a shared dialogue with service users and make them feel supported, validated and listened to.

\section{Validation and active/empathic listening}

Participants described how valuable and transformative it was when they interacted with staff who demonstrated active and empathic listening and who validated their experiences. These responses made them feel heard, cared for and supported. Such responses were reported across a range of non-specialist and specialist services:

"Accessing the mental health service and them actually saying, "I hear you. I hear that you need help." That was, yes, it was very transformative" (participant 210, female, 25-34, Black British Caribbean, London).

"Things I found helpful were, yes, people [voluntary sector service] listening, people empathising, people sympathising...People checking in, also, with what you need" (participant 101, female, 18-24, White British, London).

\section{Compassion}

Participants wanted to receive support from staff who showed a genuine interest in understanding their distress and in identifying ways to best support them as an individual:

"Having a sense that someone actually really values you and is bothered about you, rather than that they're trying to manage you in some way that they've been told is the right thing to do. So someone actually being responsive on an individual level" (participant 212, female, 45-54, White British, Yorkshire \& Humber).

\section{Open dialogue that fosters collaboration}

Participants spoke of the benefit of an open dialogue with staff and collaborative discussions about their experiences/needs so that the right support is offered:

"Instead of making an assumption based on the notes that proceeded me...she said, "I'm not going to read anything. Let me get to know you first"... what that enabled was more of a dialogue... It facilitated a change in diagnosis. It facilitated a change in direction in terms of intervention...It's such a simple thing, but it was transformative in my life" (participant 213, female, 35-44, White British, East Midlands).

Many, however, reported staff not listening: 
"It feels like I talk, and I'm not listened to, and then I'm just told, "This is what we are going to do." (participant 205, female, 35-44, British Asian, East Midlands).

"I was ignored... what she [staff at CMHT] was offering was, like, some breathing techniques. This is probably helpful for someone with lower and less complex needs than mine" (participant 103, female, White British, 25-34, South East).

\section{Consistency and continuity}

The theme Consistency and Continuity describes the benefit of service users receiving timely, consistent and continued support .

\section{Consistent and continued support from known staff}

Participants wanted services to recognise the longerterm needs of people with CEN and to address these needs by providing consistency and continuity of care. They spoke of the value of services understanding that they have ongoing needs that require support not solely during periods of crisis:

"That stability, that consistency of care, and that understanding and approach that actually this is a long-term issue...I can operate with periods of health, but that doesn't mean that it isn't really hard and that I don't need that support. As opposed to, "Oh, you know, you are doing really well at the moment. Off you pop."” (participant 213, female, 35-44, White British, East Midlands).

Yet, several participants felt they were only provided with adequate periods of care during times of mental health crisis:

"Why should I have to be constantly on a, sort of, cliff edge, or jumping off the cliff for it to be resolved, or to have some intervention?" (participant 210, female, 25-34, Black British Caribbean, London).

Some participants described receiving consistent support from a member of staff that they had time to develop a relationship with, and who continued to support them over a longer period. This experience fostered trust and led participants to feel that services understood the longer-term nature of their needs and were invested in helping them address these:

"The thing that has been helpful is that in healthier periods whilst I haven't needed the same intensity of care, having that continuity of care has kept me well, as opposed to them withdrawing and me deteriorating and then needing something more intense... definitely that stability of care, and that ability to be alongside you" (participant 213, female, 35-44, White British, East Midlands).

Some participants described the shift patterns characteristic of crisis services as incompatible with providing consistency and continuity of care:

"If you've got a mental health team coming to visit you, you see a different person every daey...you don't get a chance to build a relationship with someone... if you've got a personality disorder or complex needs, that's going to be more important than ever, to have one person to build a relationship with. Otherwise, everything is changing and that's not what you need" (participant 101, female, 18-24, White British, London).

\section{Collaborative working across services}

Participants described the benefit of agencies who provide mental health care working more collaboratively together to coordinate service users' needs:

"The main improvement would be to join things up a bit better. Some of what you need is out there, it's just that it's not connected...the Crisis Resolution Team isn't connected to my GP or to the [specialist unit]" (participant 105, female, 35-44, White British, London).

"You've got to work together, because ultimately, by working together, you get that person well faster" (participant 214, female, 25-34, White English, North East).

Many participants spoke about their experience of statutory mental health services being disjointed, with discrete interventions delivered by each service and a lack of joined-up collaborative working practices:

"I kept getting bounced backwards and forwards between different bits of this, like, very opaque system....it was a long process of being passed between different teams, and then, eventually, someone...saying "You should be treated in a different setting".. she referred me to [specialist community 'personality disorder' service]" (participant 105, female, 35-44, White British, London).

\section{Consistency of support across localities}

Participants spoke of geographical disparities in the provision of mental health services, service availability and different approaches to care:

"In the south, you know, [I've] actually seen a crisis 
house ... there's none of that in this area ... the language they use, the way the operate, it's totally different depending on which part of the country you live in" (participant 203, female, Mixed Race, North West).

\section{Timely support}

Participants spoke of the importance of being able to receive the right care at the right time, but found that high thresholds for access to some services made this difficult:

"To tell somebody in emotional pain, "Your pain is not significant for me compared to this person.... Why do I have to be so far gone to get help? (participant 210, female, 25-34, Black British Caribbean, London).

Some participants described how the tiered system required them to get a referral from one service to access another that provided more intensive mental health support. This process created delays, as service users needed to get approval for the referral from the first service and then wait for the documentation to be processed and approved by the second:

"The whole referral process [to a specialist community 'personality disorder' service] took six months... I was very, very mentally unwell...but unfortunately it felt like the policies and procedures were above everything else...it was very frustrating" (participant 104, female, 25-34, White British, Yorkshire $\mathcal{E}$ Humber).

\section{Adaptability and accessibility}

The theme Adaptability and Accessibility describes ways in which services can promote service user engagement and adapt to their needs over time.

\section{Proactive engagement with service users}

Participants reflected on the benefit of services taking measures to encourage engagement, through proactive steps to connect with service users:

"With this complex needs service, there is recognition that there may be many reasons why people aren't turning up...They will say, "Why didn't you come? What were the problems? How can I help you overcome those?" (participant 209, female, 45-54, White English, West Midlands).

Some participants spoke of cathartic and healing experiences of receiving support from peer workers and called for peer-support roles to become formalised roles of employment and for peer-workers to receive skillsbased training:

"I think the best people are people that have lived experience of mental health. Because...they can actually say, "I understand", or, "I've been there,... and they mean it, it's true" (participant 111, male, 55-64, White Other, London).

"I feel very strongly that there needs to be...professionalisation of peer support...It is actually about really valuing the experiences of somebody with lived experience...it needs to be done in a framework of recompense for the work and appreciation of the role...I think it is really important, but I think it needs to be valued appropriately and invested in appropriately" (participant 213, female, 35-44, White British, East Midlands).

\section{Flexibility in service delivery}

Participants spoke of the benefit of services being flexible in their approach to care to ensure that service users receive the right level of support at the right time. This included flexibility with respect to the types of support/ intervention offered, with services having the ability to connect service users to different forms of support when needed, and in the ability of services to provide greater input at times when service users demonstrate greater need for support. These responses led to improvements in their well-being:

"[Service was] flexible around if I wasn't getting on with a worker or if something just wasn't right. I was getting CBT therapy for a while that wasn't particularly right, and they referred me onto a psychodynamic psychological therapy. So, they were really good at being flexible" (participant 104, female, 25-34, White British, Yorkshire E Humber).

"She said [AEE psychiatric liaison], "Look, I'm going to give up my time, and you are going to come and see me here, at this hospital ...I want to help you... let's make something happen..... She ended up seeing me...10 weeks in total, that was the beginning not just [of] me not trying to kill myself, but...the complete turnaround of my life was kickstarted by the positivity of just someone making that effort on my part" (participant 214, female, 25-34, White English, North East).

\section{Ability to offer a range of interventions}

Participants wanted services to facilitate their access to a range of forms of support. However, many explained that 
non-specialist services had limited knowledge of the support available for people with CEN or an understanding of the types of support that would be most appropriate for them:

\begin{abstract}
"The whole system needs to be much more connected and people [say], "Okay, well, I know what I can help with and I know what I can't help with.....so everybody in the system is like, "Okay, this isn't technically what I can help you with, but this person can." (participant 101, female, 18-24, White British, London).
\end{abstract}

"I'm becoming aware that there're quite a lot of different tools out there that might work differently for different people at different times. Having the facility to actually, maybe, play around with those and experiment...rather than, "This is all that's on offer, get on with it." (participant 212, female, 45-54, White British, Yorkshire \& Humber).

Participants recognised that the ability of services to deliver the good care practices outlined in the themes Consistency and Continuity and Adaptability and Accessibility were impacted by resourcing issues, service funding, staffing levels and staff training.

\section{Discussion}

Participants reported a range of experiences of community practice from" abusive, damaging" to "phenomenal". Although participants reported several examples of positive care, many reported a range of negative experiences related to severe stigmatisation, a lack of staff knowledge and understanding of their intersecting needs/experiences, limited effective support and service fragmentation. Relational Practice was identified as the best way to support service users in the community, and participants reported elements of this practice across both specialist and non-specialist community services.

Relational practice theories are present in psychological, psychiatric, nursing and social work practices [3033] and describe how personal, interpersonal and social structural factors shape a person's lived experience [34]. This study provides further insight into this theory by explaining how personal, interpersonal and social structural factors interact and shape people's experiences of care. Indeed, our overarching theme of relational practice includes four sub-themes: (1) Understanding; (2) Interpersonal Connection; (3) Consistency and Continuity; (4) Adaptability and Accessibility. These four sub-themes are inter-connected and describe how staff and service practices/structures can work in a way that creates positive experiences for service users and leads to improved service user outcomes. Relational practice comprises staff delivering care in a non-stigmatising, individualised and compassionate way, and delivering care that is traumainformed (sub-themes Understanding and Interpersonal Connection). When staff work holistically and collaboratively with service users to coordinate support for their complex needs (sub-themes Understanding and Consistency and Continuity). When service structures allow for flexibility and continuity of care, accommodate the ongoing and changing nature of service users' needs, and implement joint-working practices with other services (sub-themes Interpersonal Connection, Consistency and Continuity and Adaptability and Accessibility).

\section{Understanding \\ Inclusive, non-judgemental, and non-discriminatory approaches}

A key finding of this study is the need for services to adopt inclusive, non-judgemental and non-discriminatory approaches when supporting people with CEN. Participants reported numerous experiences of stigmatising attitudes from service providers regarding the diagnostic label 'personality disorder' and how it is often perceived, including being seen as someone who cannot be helped, being de-personalised or being viewed as a potential trouble-maker. There is evidence to suggest that people with CEN experience more stigma than people with other mental health diagnoses [35]. Stigmatisation can result in iatrogenic harm when service providers dismiss peoples experience of distress, refuse them access to services or fail to demonstrate compassion in the therapeutic relationship [14]. Generally, participants in this study who received support from specialist community 'personality disorder' services felt less stigmatised and judged about their mental health needs. This may reflect a better trained workforce with regards to knowledge and understanding of CEN [1].

Despite a body of literature evidencing the extent and impact of stigmatisation on people with CEN, few wide scale coordinated efforts have been undertaken to challenge these attitudes and practices. Our related qualitative work with community staff highlights that stigmatising views and behaviours arise not simply due to a lack of knowledge but also due to factors such as staff workloads, confidence and competencies in managing the multiple and longer-term needs of service users, as well as in managing issues of risk (Foye et al., submitted). There is an urgent need for research to identify the origins of these stigmatising behaviors and evaluations of programmes that seek to challenge and change stigmatising views and behaviours among staff. The Knowledge and Understanding Framework programme is an example of a training programme that has been shown to improve staff competencies in supporting service users with CEN [36]. 
Participants in this study also described how their intersecting identities (e.g. ethnicity, sexuality, age, class, physical appearance) can influence their mental health wellbeing and lead them to experience multiple forms of stigmatisation. Yet, participants reported that community services often demonstrated a lack of awareness of these issues or indeed perpetuated these forms of stigma. Across a range of healthcare settings, people are found to experience intersecting forms of stigma related to their different identities, which negatively impact on their physical and mental health [37]. However, there remains a lack of research on the impact intersecting stigma experiences among people with mental health problems [38]. There is some research describing how experiences of racism adversely impact on the ability of services to foster communication and trust, and in delivering effective care for Black and minority ethnic service users [39-41] which can lead to minority groups disengaging from mental health service use [42]. There is also some research which shows that LGBTQ+ groups can experience a lack of understanding from staff when accessing healthcare [43], which our study provides further evidence of.

\section{Holistic approaches}

Our study adds further evidence to support our qualitative meta-synthesis finding that a holistic approach to care is of central importance to service users, and an overriding focus on the provision of psychological interventions targeting self-harm can neglect many current challenges that service users are dealing with [1]. Participants in this study described wanting help with a range of social and occupational needs (e.g. housing, benefits, employment, social connections) alongside their mental health needs. They explained that a failure of services to recognise and address their competing social needs often meant they were offered forms of support that were either insufficient or with which they could not engage. Our review paper on models of care for people with CEN and the NICE guidelines for people with 'borderline personality disorder' also underline the need for interventions to extend beyond just psychological therapies, to incorporate support for social and practical needs [23]. Despite a well-established relationship between social well-being and mental health, existing research and mental health service guidelines predominately focus on the provision of psychological and pharmacological interventions [44]. Further research would, therefore, benefit from reviewing the international evidence-base on integrated mental health and social need interventions and their respective strengths and weaknesses in improving service user experiences and outcomes. Research should also focus on effective implementation strategies for evidence-based holistic interventions, which represent a key activity in achieving parity of esteem (compared to physical health care) for mental health care $[45,46]$.

With respect to specialist community 'personality disorder' services, numerous evaluations [47-53] have highlighted the benefit that these services provide through delivery of holistic support (i.e. psychological, social, occupational interventions) [48, 52, 54, 55], which can improve clinical outcomes and service satisfaction among service users [47, 49-51], and reduce further health service use costs [56]. It is important to note, however, that the uptake of specialist community 'personality disorder' services remains limited and highly varied. In addition, there is considerable variation in the approach that generic mental health services have taken to meet the needs of service users with CEN [57]. What is clear, however, is that policy [58] and healthcare guidelines [55] steer organizations to adopt a strategy which ensures both the availability of effective generic and specialist service interventions. The role of specialist services being to support generic services and to provide specialized interventions for those with the most severe conditions and those most at risk [59]. Our findings support calls for generic mental health services to increase availability of holistic interventions [60,61], and there are now novel CEN-specific holistic psychological interventions being evaluated in generic mental health services which are showing strong clinical promise $[61,62]$.

\section{Trauma-informed care}

Participants described the benefit of community services adopting trauma-informed care (TIC), which acknowledges peoples' experiences of trauma and adopts practices that promote peoples' safety, empowerment and choice. TIC is an organisational approach that recognises the impact of trauma on an individual's life and their ability to engage with and use health services [63]. TIC approaches comprise four key components: (1) realizing the high prevalence of trauma and how it affects individuals, groups and organisations, (2) recognising the signs of trauma and how it affects all individuals within an organisation, (3) applying TIC to all areas of functioning in an organisation, (4) operating in a way that prevents re-traumatisation [64]. There are increasing calls by people with relevant lived experience, clinicians, researchers and policy makers for mental health services to adopt trauma-informed care (TIC) practices [19, 30, 64, 65]. For example, the Power Threat Meaning Framework published by the British Psychological Society seeks to understand mental distress from a social, cultural, psychological and biological approach, replacing medicalised questions like "what is wrong with you?" to "what has happened to you?" It also acknowledges the centrality 
of the relational context in decisions about mental health need [66]. Successful TIC approaches require responses at both organisational and individual staff levels [64]. Organisational activities such as engaging service users in service design, hiring trauma-informed staff (i.e. people that adopt language and behaviours that take in to consideration trauma experiences among service users and colleagues) and training all staff in TIC practice, alongside staff implementation of routine enquiry about trauma, working collaboratively with service users in care planning, and knowledge of trauma-based support services are critical [67]. To date, there is a paucity of research examining the success of TIC training programmes in improving mental health staff knowledge, attitudes and behaviours around TIC. The few studies that exist report positive changes to staff knowledge and behaviours immediately after training and over the short-term $[36,68,69]$. More work is needed to examine how mental health services can support staff in sustaining positive TIC training outcomes over the longer-term (e.g. via clinical supervision, reflective practice). More crucially, there is a pressing need to evaluate whether the positive gains staff report following TIC training lead to improved outcomes and higher service satisfaction among service users. A recent UK survey of mental health professionals and other healthcare and community service professionals examined barriers to the adoption of TIC across their settings [70]. Respondents highlighted that the biggest barriers to adoption were that experiences of trauma are often medicalised and that services are too piecemeal to meet the needs of trauma survivors. Further research is, therefore, needed to identify how best to address these barriers to implementation of TIC approaches in mental health care settings.

\section{Interpersonal connection}

We found that service users want to be supported by staff who treat them with respect and compassion, who listen with empathy and who validate their individual experiences. These findings are supported by the wider qualitative literature on service users with CEN [1], and staff themselves also report the importance of these values and characteristics in their care of service users [2]. These experiences can foster trust between service users and staff, critical for establishing and maintaining the therapeutic relationship [71].

Participants also want open dialogue and collaboration with staff to facilitate fuller disclosure of their experiences and needs and to ensure appropriate care is provided. Recent psychiatric guidelines on best practice approaches for people with CEN advocate for care management plans that are co-constructed by staff and service users, to ensure they fully reflect and address service users' biological, psychological and social needs [19]. Shared decision making is another approach that allows service users and staff to make joint healthcare decisions, and is found to improve service user outcomes [72]. A recent review of shared decision making found that medical and diagnostic models of working in mental health services can result in staff not acknowledging service users' expertise, and create power imbalances with service users feeling they are informed rather than involved in the care they receive [73].

\section{Consistency and continuity}

Participants wanted community services to acknowledge the long-term nature of CEN and to provide service users with consistent, ongoing support from staff. The provision of intensive psychological therapies (e.g. dialectical behavioural therapy [74]) was beneficial to several participants but this intervention alone was not sufficient in addressing all of participants' changing and longer-term needs. Participants identified that the delivery of longerterm support and consistency of service provision across different regions is often compromised by a lack of sufficient funding for services. This reflection aligns with the experience of clinicians, who report that a focus on avoiding dependency in the care relationship has encouraged the delivery of discrete interventions designed to promote rapid movement through and out of the mental health system, which is unsuitable for service users with enduring needs [30]. Our related qualitative review exploring staff experiences in supporting people with CEN has identified a concern among some staff that offering sustained support can make the ending of an intervention more challenging for service users [2]. Yet, participants in this study reported that it is the absence of longer-term support which is particularly challenging. Further research would benefit from working with service users and staff to jointly identify best practice continuity of care approaches.

\section{Adaptability and accessibility}

Participants want services to be flexible with respect to the extent and type of support they offer service users, in response to their changing needs at any given time. This finding and those outlined above underline the benefit of flexible services structures which allow people to transition between more intensive and less intensive periods of support in a timely manner whilst also continuing to receive assistance for their ongoing needs. Participants also want staff to facilitate their engagement with services. Participants reflected that the ability of services to deliver these types of care is often compromised by a lack of sufficient funding, resulting in limited intervention options and limited staff capacity to work flexibly and 
proactively engage service users. Increased equity in service provision for people with CEN, compared to people with other mental health needs, would go some way to redress these limitations.

Several participants also spoke of the value in services establishing peer support roles. Peer support approaches are increasing within mental health care settings but there is an absence of high-quality evaluations on the effectiveness of these models in improving service user outcomes [75] and warnings from some commentators that the formalisation of peer roles may threaten the equality and independence of this approach which can be a key to its success [76]. Alongside the need to formally test peer support interventions of high methodological quality, further research could explore how peer support can best fit within services that model the relational practice approaches outlined above (e.g. individualised, holistic and compassionate care that includes collaborative care planning and consistent and flexible support over time). Peer workers and lived experience practitioners are increasingly working alongside generic community mental health services to improve responses to CEN and these activities would benefit from formal evaluation to explore its impact on service operations, staff practice and service user outcomes.

\section{Relational practice}

Our study's overarching theme identifies a relational practice approach as the best way for community services to support service users with CEN. There are increasing calls for mental health services to adopt relational practice approaches, which prioritise the development and maintenance of therapeutic relationships over standardised procedures [30]. Yet, it is argued that relational approaches may conflict with dominant managerialism practices [32] common in healthcare settings, as the latter emphasises implementation of standardised procedures and measurement of success against restricted performance targets [77]. As a result, the importance of relational work in improving outcomes and care experiences can be downgraded. This study highlights the importance of relational work in improving service users' experience of care and their outcomes. Other research supports this finding, with interpersonal components identified as key elements of evidenced-based interventions for CEN [78].The ability of staff to work relationally is shaped by organisational factors such as staffing ratios, supportive peer/management relationships [31] and opportunities for reflective practice and clinical supervision [19]. In addition, an understanding at an organisational-level that relational work requires skills and expertise which are not simply innate characteristics of individuals [79]. To successfully deliver relational practice, service design and intervention development must be co-produced with people with relevant lived experience, their carers and the professionals who support them.

\section{Strengths and limitations}

A group of experts by experience and occupation coproduced this study. In addition, most research interviews and analyses were carried out by lived experience researchers. We were successful in interviewing people with a broad range of experiences of service use with respect to specialist and non-specialist services, current and previous service use, service use over many years versus minimal use, including those who intentionally disengaged from service use after negative experiences. We did our best to diversify the study sample and 12/30 participants had ethnic backgrounds which are in the minority in England. There is a lack of research in this field with minority ethnic communities and this paper provides some valuable first-hand accounts. We also achieved a good geographical spread of participants; the East of England is the only region from which we had no representation.

Despite our broad inclusion criteria, all participants had received some sort of 'personality disorder' diagnosis at some time and we did not interview anyone who felt that they had been denied access to services because their CEN went undetected. We cannot, therefore, give any insight regarding the experiences of people who may have relevant needs, but do not access services or receive a diagnosis. Only a few men took part in the study $(6 / 30)$. We asked participants about a broad range of community services, including voluntary sector services, but their views were largely based on their experiences of the NHS. Our recruitment methods means that we have not captured the experiences of people who do not use social media or relevant CEN networks.

\section{Conclusions}

This study identifies how relational practice approaches have the potential to improve community care for service users with CEN. Further research and service development is now needed and must be co-produced with people with relevant lived experience, their carers and the professionals who support them, to examine how best to implement relational practice approaches across the mental health service system.

\section{Lived experience commentary}

This qualitative study reports significant difficulties in accessing appropriate community services for CEN which cast doubt on what progress, if any, has actually been made in this area since publication of the 2003 seminal document, "Personality Disorder: No Longer 
a Diagnosis of Exclusion" [80]. We note the disturbing irony that the commissioning of this research has happened at a time when the coercive Serenity Integrated Mentoring (SIM) programme [81], which threatens to criminalise people with CEN in crisis and withholds essential mental and physical health care [82], has been adopted so readily across mental health trusts in England without proper scrutiny or evidence base.

When interviewing for this study, we were particularly struck by the recurrent accounts of stigmatising attitudes amongst staff in community mental health services. Encountering stigma in this context can cause profound iatrogenic harm and tackling this pervasive and enduring stigma must be a key priority for future service improvement. The paper's overarching theme and recommendation of relational practice - a collaborative framework for providing care - may contribute to a sound foundation for addressing this.

Such collaboration in individual care reflects the need for staff to embrace true co-production at all stages from design to delivery of any services for people with CEN. However, that will require a substantial culture change in many services where the reality of co-production initiatives often falls far short of its guiding principles [83]. Likewise, embedding lived experience practitioners at all levels of seniority will be indispensable for meaningful change, but this is often still met with great resistance from staff who fear their status being challenged.

The findings also highlight the need to acknowledge and address intersecting challenges of trauma, inequalities and discrimination in a more holistic approach. We suggest that future research and policy work need to go further than the commission for this project allowed, listen to the experiential knowledge of service users in this study and the project's January 2019 workshop [84], and abandon the pejorative construct of the disordered personality which fuels stigmatising attitudes. There is an urgent need to develop approaches focused around what has happened to the person in distress and supporting them with their natural reactions to trauma without judgement or prejudice.

Eva Broeckelmann and Stephen Jeffreys.

\section{Abbreviations \\ CEN: Complex emotional needs; LGBTQ+: Lesbian, gay, bisexual, transgender, queer/questioning people.}

\section{Acknowledgements}

We thank all of the service users who participated in this study and shared their experiences with us. We wish to thank all members of our study working group for their continued guidance and support throughout this project.

\section{Authors' contributions}

$K T, R S, E B, S J e, T J, D A, J R, J B, M J C, R H, S M, V N, A S, B L E, S J O, S O$ co-produced the study protocol and interview topic guide. EB, SJe, DA, JR, RS, KT, UF led the data analyses and all authors conducted interpretation of the data. KT, RS, JO wrote the manuscript and EB, SJe, TJ, DA, JR, JB, MJC,OD, RH, PM, SM, VN, UF, AS, BLE, SJo, SO critically reviewed and improved the manuscript. All authors have approved the manuscript.

\section{Funding}

This paper presents independent research commissioned and funded by the National Institute for Health Research (NIHR) Policy Research Programme, conducted by the NIHR Policy Research Unit (PRU) in Mental Health. The views expressed are those of the authors and not necessarily those of the NIHR, the Department of Health and Social Care or its arm's length bodies, or other government departments.

\section{Availability of data and materials}

The data that support the findings of this study are available on request from the corresponding author (KT). The data are not publicly available due to the sensitive nature of the data.

\section{Declarations}

\section{Ethics approval and consent to participate}

Ethical approval for the study was obtained from King College London's PNM Research Ethics Subcommittee on 16/05/2019, reference HR-18/19-10795, and all methods performed in accordance with the approval regulations. Informed consent was obtained from all study participants.

\section{Consent for publication}

Not Applicable.

\section{Competing interests}

The authors declare that they have no competing interests.

\section{Author details}

${ }^{1}$ Health Service and Population Research Department, NIHR Mental Health Policy Research Unit, Institute of Psychiatry, Psychology \& Neuroscience, King's College London, London, UK. ²David Goldberg Centre, Institute of Psychiatry, Psychology \& Neuroscience Kings College London, De Crespigny Park, Denmark Hill, Room H3.06, London SE5 8AF, UK. ${ }^{3}$ Implementation Science, Health Service and Population Research Department, Institute of Psychiatry, Psychology \& Neuroscience, King's College London, London, UK. ${ }^{4}$ National Institute for Health Research (NIHR) Applied Research Collaboration, South London, London, UK. ${ }^{5} \mathrm{NIHR}$ Mental Health Policy Research Unit Complex Emotional Needs Lived Experience Working Group, Health Service and Population Research Department, Institute of Psychiatry, Psychology \& Neuroscience, King's College London, London, UK. ${ }^{6}$ Division of Psychiatry, NIHR Mental Health Policy Research Unit, University College London, London, UK. ${ }^{7}$ Division of Psychiatry, Imperial College London, London, UK. ${ }^{8}$ West London Mental Health Trust, London, UK. ${ }^{9}$ Berkshire Healthcare NHS Foundation Trust, Bracknell, UK. ${ }^{10}$ Bristol Medical School, University of Bristol, Bristol, UK. ${ }^{11}$ Camden and Islington NHS Foundation Trust, London, UK. ${ }^{12}$ Health Service and Population Research, Institute of Psychiatry, Psychology \& Neuroscience, and Florence Nightingale Faculty of Nursing, Midwifery \& Palliative Care, King's College London, London, UK.

Received: 21 June 2021 Accepted: 9 November 2021

Published online: 27 January 2022

\section{References}

1. Sheridan Rains L, Echave A, Rees J, Scott HR, Lever-Taylor B, Broeckelmann $E$, et al. Service user experiences of community services for complex emotional needs: a qualitative thematic synthesis. PLOS ONE. 2021;16(4): e0248316.

2. Troup J, Lever-Taylor B, Rains LS, Broeckelmann E, Russell J, Jeynes T, et al. Clinician perspectives on what constitutes good practice in community services for people with complex emotional needs: a qualitative thematic meta-synthesis. medRxiv. 2020. 
3. Campbell K, Clarke KA, Massey D, Lakeman R. Borderline personality disorder: to diagnose or not to diagnose? That is the question. Int J Ment Health Nurs. 2020;29(5):972-81.

4. Klein P, Fairweather AK, Lawn S, Stallman H, Cammell P. Structural stigma and its impact on healthcare for consumers with borderline personality disorder: protocol for a scoping review. 2021.

5. Ussher JM. Diagnosing difficult women and pathologising femininity: gender bias in psychiatric nosology. Fem Psychol. 2013;23(1):63-9.

6. Ring D, Lawn S. Stigma perpetuation at the interface of mental health care: a review to compare patient and clinician perspectives of stigma and borderline personality disorder. J Ment Health. 2019;12:1-21.

7. Winsper C, Bilgin A, Thompson A, Marwaha S, Chanen AM, Singh SP, et al. The prevalence of personality disorders in the community: a global systematic review and meta-analysis. Br J Psychiatry. 2019;216(2):69-78.

8. Beckwith H, Moran PF, Reilly J. Personality disorder prevalence in psychiatric outpatients: a systematic literature review. Personal Ment Health. 2014;8(2):91-101.

9. Moran P, Jenkins R, Tylee A, Blizard R, Mann A. The prevalence of personality disorder among UK primary care attenders. Acta Psychiatr Scand. 2000;102(1):52-7.

10. Crawford MJ, Rushwaya T, Bajaj P, Tyrer P, Yang M. The prevalence of personality disorder among ethnic minorities: findings from a national household survey. Personal Ment Health. 2012;6(3):175-82.

11. Tyrer P, Reed GM, Crawford MJ. Classification, assessment, prevalence, and effect of personality disorder. Lancet. 2015;385(9969):717-26.

12. Hossain A, Malkov M, Lee T, Bhui K. Ethnic variation in personality disorder: evaluation of 6 years of hospital admissions. BJPsych Bull. 2018;42(4):157-61.

13. Evans S, Sethi F, Dale O, Stanton C, Sedgwick R, Doran M, et al. Personality disorder service provision: a review of the recent literature. Ment Health Rev J. 2017:22(2):65-82

14. Sheehan L, Nieweglowski K, Corrigan P. The stigma of personality disorders. Current Psychiatry Rep. 2016;18(1):11.

15. Markham D, Trower P. The effects of the psychiatric label 'borderline personality disorder'on nursing staff's perceptions and causal attributions for challenging behaviours. Br J Clin Psychol. 2003;42(3):243-56.

16. Lewis $G$, Appleby L. Personality disorder: the patients psychiatrists dislike. Br J Psychiatry. 1988;153(1):44-9.

17. Chanen A, Sharp C, Hoffman P, for Prevention GA. Prevention and early intervention for borderline personality disorder: a novel public health priority. World Psychiatry. 2017;16(2):215.

18. Flynn S, Graney J, Nyathi T, Raphael J, Abraham S, Singh-Dernevik S, et al. Clinical characteristics and care pathways of patients with personality disorder who died by suicide. BJPsych Open. 2020;6(2).

19. Royal College of Psychiatrists. Services for people diagnosable with personality disorder. London: Royal College of Psychiatrists; 2020.

20. Simonsen S, Bateman A, Bohus M, Dalewijk HJ, Doering S, Kaera A, et al. European guidelines for personality disorders: past, present and future. Borderline Pers Disord Emotion Dysregulation. 2019;6(1):1-10.

21. England NHS. The NHS long term plan. London: NHS England; 2019

22. Ng F, Townsend ML, Jewell M, Marceau EM, Grenyer BF. Priorities for service improvement in personality disorder in Australia: perspectives of consumers, carers and clinicians. Personal Ment Health. 2020;14(4):350-60.

23. Storebø OJ, Stoffers-Winterling JM, Völlm BA, Kongerslev MT, Mattivi JT, Jørgensen MS, et al. Psychological therapies for people with borderline personality disorder. Cochrane Database Syst Rev. 2020(5):CD012955:1-512.

24. Booth A, Hannes K, Harden A, Noyes J, Harris J, Tong A. COREQ (consolidated criteria for reporting qualitative studies). Guidelines for reporting health research: a user's manual. 2014:214-26.

25. National Institute for Health Research. Guidance on co-producing a research project. London: National Institute for Health Research; 2021.

26. Beresford P, Farr M, Hickey G, Kaur M, Ocloo J, Tembo D, et al. COVID-19 and Co-production in Health and Social Care Research, Policy, and Practice. Volume 1:The Challenges and Necessity of Co-production. Bristol: Policy Press; 2021

27. Tembo D, Hickey G, Montenegro C, Chandler D, Nelson E, Porter K, et al. Effective engagement and involvement with community stakeholders in the co-production of global health research. BMJ. 2021;372:1-6.
28. NVivo (Version 12) [Internet]. QSR International Pty Ltd. 2018. Available from: https://www.qsinternational.com/nvivo-qualitative-data-analysissoftware/home.

29. Sweeney A, Clement S, Filson B, Kennedy A. Trauma-informed mental healthcare in the UK: what is it and how can we further its development? Ment Health Rev J. 2016;21(3):174-92.

30. Dale $\mathrm{O}$, Haigh $\mathrm{R}$, Blazdell J, Sethi F. Social psychiatry, relational practice and learning from COVID-19. Ment Health Rev J. 2020;25(4):297-300.

31. Doane $\mathrm{GH}$, Varcoe C. Relational practice and nursing obligations. Adv Nurs Sci. 2007;30(3):192-205.

32. Ingram R, Smith M. Relationship-based practice: emergent themes in social work literature. 2018.

33. Tufekcioglu S, Muran JC. A relational approach to personality disorder and alliance rupture. Integrated treatment for personality disorders New York: Guilford. 2014

34. Zou P. Relational practice in nursing: a case analysis. Nurs Health Care. 2016;1(1):1-5.

35. Rao H, Mahadevappa H, Pillay P, Sessay M, Abraham A, Luty J. A study of stigmatized attitudes towards people with mental health problems among health professionals. J Psychiatr Ment Health Nurs. 2009;16(3):279-84.

36. Davies J, Sampson M, Beesley F, Smith D, Baldwin V. An evaluation of knowledge and understanding framework personality disorder awareness training: can a co-production model be effective in a local NHS mental health trust? Personal Ment Health. 2014;8(2):161-8.

37. Turan JM, Elafros MA, Logie CH, Banik S, Turan B, Crockett KB, et al. Challenges and opportunities in examining and addressing intersectional stigma and health. BMC Med. 2019;17(1):1-15.

38. Oexle N, Corrigan PW. Understanding mental illness stigma toward persons with multiple stigmatized conditions: implications of intersectionality theory. Psychiatr Serv. 2018;69(5):587-9.

39. Holley LC, Tavassoli KY, Stromwall LK. Mental illness discrimination in mental health treatment programs: intersections of race, ethnicity, and sexual orientation. Community Ment Health J. 2016;52(3):311-22.

40. Delphin-Rittmon ME, Flanagan EH, Andres-Hyman R, Ortiz J, Amer MM, Davidson L. Racial-ethnic differences in access, diagnosis, and outcomes in public-sector inpatient mental health treatment. Psychol Serv. 2015;12(2):158.

41. Gabbidon J, Farrelly S, Hatch SL, Henderson C, Williams P, Bhugra D, et al. Discrimination attributed to mental illness or race-ethnicity by users of community psychiatric services. Psychiatr Serv. 2014;65(11):1360-6.

42. Richman LS, Kohn-Wood LP, Williams DR. The role of discrimination and racial identity for mental health service utilization. J Soc Clin Psychol. 2007;26(8):960-81.

43. Kattari SK, Walls NE, Whitfield DL, Langenderfer-Magruder L. Racial and ethnic differences in experiences of discrimination in accessing health services among transgender people in the United States. Int J Transgenderism. 2015;16(2):68-79.

44. Johnson S. Social interventions in mental health: a call to action. Soc Psychiatry Psychiatr Epidemiol. 2017;52:245-7.

45. Bailey S, Thorpe L, Smith G. Whole-person care: from rhetoric to reality. In: Achieving parity between mental and physical health. London: Royal College of Psychiatrists; 2013.

46. Mitchell AJ, Hardy S, Shiers D. Parity of esteem: addressing the inequalities between mental and physical healthcare. BJPsych Adv. 2017;23(3):196-205

47. Chiesa M, Sharp R, Fonagy P. Clinical associations of deliberate self-injury and its impact on the outcome of community-based and long-term inpatient treatment for personality disorder. Psychother Psychosom. 2011:80(2):100-9.

48. Crawford M, Rutter D. Lessons learned from an evaluation of dedicated community-based services for people with personality disorder. Ment Health Rev J. 2007;12(4):55-61.

49. Jones B, Juett G, Hill N. Initial outcomes of a therapeutic communitybased outpatient programme in the management of personality disorder. Therapeutic communities: the international journal of therapeutic. Communities. 2013;34(1):41-52.

50. Miller S, Crawford MJ. Open access community support groups for people with personality disorder: attendance and impact on use of other services. Psychiatrist. 2010;34(5):177-81 
51. Pearce S, Scott L, Attwood G, Saunders K, Dean M, De Ridder R, et al. Democratic therapeutic community treatment for personality disorder: randomised controlled trial. Br J Psychiatry. 2017;210(2):149-56.

52. Wilson L, Haigh R. Innovation in action. 2011.

53. Chiesa M, Fonagy P, Gordon J. Community-based psychodynamic treatment program for severe personality disorders: clinical description and naturalistic evaluation. J Psychiatr Pract. 2009;15(1):12-24

54. Price K, Gillespie S, Rutter D, Dhillon K, Gibson S, Faulkner A, et al. Dedicated personality disorder services: a qualitative analysis of service structure and treatment process. J Ment Health. 2009;18(6):467-75.

55. National Institute for health and care excellence. Borderline personality disorder: recognition and management. London; 2009.

56. Kane E, Reeder N, Keane K, Prince S. A cost and economic evaluation of the Leeds personality disorder managed clinical network - a service and commissioning development initiative. Personal Ment Health. 2016;10(3):169-80.

57. Dale O, Sethi F, Stanton C, Evans S, Barnicot K, Sedgwick R, et al. Personality disorder services in England: findings from a national survey. BJPsych Bull. 2017:41(5):247-53.

58. Royal College of Psychiatrists. Services for people diagnosable with personality disorder: position statement. London: Royal College of Psychiatrists; 2020 .

59. National Institute for Mental Health for England. Personality Disorder: no Longer a Diagnosis of Exclusion. In: Policy Implementation Guidance for the Development of Services for People with Personality Disorder, Gateway Reference 1055. London: NIMH(E); 2003.

60. Roughley M, Maguire A, Wood G, Lee T. Referral of patients with emotionally unstable personality disorder for specialist psychological therapy: why, when and how? BJPsych Bull. 2021;45(1):52-8.

61. Crawford MJ, Thana L, Parker J, Turner O, Xing KP, McMurran M, et al. Psychological support for personality (PSP) versus treatment as usual: study protocol for a feasibility randomized controlled trial of a low intensity intervention for people with personality disorder. Trials. 2018;19(1):1-13.

62. Crawford MJ, Thana L, Parker J, Turner O, Carney A, McMurran M, et al. Structured psychological support for people with personality disorder: feasibility randomised controlled trial of a low-intensity intervention. BJPsych Open. 2020;6e25:1-9.

63. Harris ME, Fallot RD. Using trauma theory to design service systems. Jossey-Bass/Wiley. 2001.

64. Substance Abuse and Mental Health Services Administration. SAMHSA's concept of trauma and guidance for a trauma-informed approach. Rockville, MD: Substance Abuse and Mental Health Services Administration; 2014.

65. Johnstone L, Boyle M, Cromby J, Dillon J, Harper D, Kinderman P, et al. The power threat meaning framework: towards the identification of patterns in emotional distress, unusual experiences and troubled or troubling behaviour, as an alternative to functional psychiatric diagnosis. Leicester: British Psychological Society; 2018.

66. Johnstone L, Boyle M, Cromby J, Dillon J, Harper D, Kinderman P, et al. The power threat meaning framework: overview. Leicester: British Psychological Society; 2018.

67. Menschner C, Maul A. Key ingredients for successful trauma-informed care implementation: Center for Health Care Strategies, Incorporated Trenton; 2016.

68. Williams TM, Smith GP. Does training change practice? A survey of clinicians and managers one year after training in trauma-informed care. J Ment Health Train Educ Pract. 2017;12(3):188-98.

69. Palfrey N, Reay RE, Aplin V, Cubis JC, McAndrew V, Riordan DM, et al. Achieving service change through the implementation of a traumainformed care training program within a mental health service. Community Ment Health J. 2019;55(3):467-75.

70. Trauma Informed Community of Action. Trauma-informed Community of Action Brief evaluation of the community and its use of the FutureNHS platform. North East and North Cumbria: NHS England \& Improvement; 2020.

71. Langley $\mathrm{G}, \mathrm{Klopper} \mathrm{H}$. Trust as a foundation for the therapeutic intervention for patients with borderline personality disorder. J Psychiatr Ment Health Nurs. 2005;12(1):23-32.

72. Huang C, Plummer V, Lam L, Cross W. Perceptions of shared decisionmaking in severe mental illness: an integrative review. J Psychiatr Ment Health Nurs. 2020;27(2):103-27.
73. Castillo H, Ramon S. "Work with me": service users' perspectives on shared decision making in mental health. Ment Health Rev J. 2017;22(3):166-78.

74. Linehan MM. Dialectical behavior therapy for borderline personality disorder: theory and method. Bull Menn Clin. 1987;51(3):261.

75. Lloyd-Evans B, Mayo-Wilson E, Harrison B, Istead H, Brown E, Pilling S, et al. A systematic review and meta-analysis of randomised controlled trials of peer support for people with severe mental illness. BMC Psychiatry. 2014;14(1):1-12.

76. Faulkner A, Basset T. A long and honourable history. J Ment Health Train Educ Pract. 2012;7(2):53-59.

77. Carlisle Y. Complexity dynamics: managerialism and undesirable emergence in healthcare organizations. J Med Mark. 2011;11(4):284-93.

78. Bateman AW, Gunderson J, Mulder R. Treatment of personality disorder. Lancet. 2015;385(9969):735-43.

79. Carlson $\mathrm{JH}$, Crawford M. Perceptions of relational practices in the workplace. Gender Work Organ. 2011;18(4):359-76.

80. National Institute for Mental Health in England. Personality disorder: no longer a diagnosis of exclusion. London: National Institute for Mental Health in England; 2003.

81. Jennings P, Matheson-Monnet CB. Multi-agency mentoring pilot intervention for high intensity service users of emergency public services: the Isle of Wight integrated recovery Programme. J Criminol Res Policy Pract. 2017;3(2):105-18.

82. StopSIM Coalition. Coalition Statements (StopSIM Coalition Consensus Statement relating to The High Intensity Network (HIN) and Serenity Integrating Mentoring (SIM), 21/04/21): StopSIM Coalition,; 2021 [Available from: https://www.stopsim.co.uk/.

83. Slay J, Stephens L. Co-production in mental health: a literature review, vol. 4. London: New economics foundation; 2013.

84. National Institute for Health Research Mental Health Policy Research Unit. Community 'Personality Disorder'Services Research Workshop 2019 [Available from: https://www.ucl.ac.uk/psychiatry/research/nihr-mentalhealth-policy-research-unit/past-events/community-personality-disor der-services.

\section{Publisher's Note}

Springer Nature remains neutral with regard to jurisdictional claims in published maps and institutional affiliations.

Ready to submit your research? Choose BMC and benefit from:

- fast, convenient online submission

- thorough peer review by experienced researchers in your field

- rapid publication on acceptance

- support for research data, including large and complex data types

- gold Open Access which fosters wider collaboration and increased citations

- maximum visibility for your research: over $100 \mathrm{M}$ website views per year

At BMC, research is always in progress.

Learn more biomedcentral.com/submissions 